\title{
SELECTED PROBLEMS OF SENSITIVITY AND RELIABILITY OF A JACK-UP PLATFORM
}

\author{
Bogdan Rozmarynowski \\ Tomasz Mikulski \\ Gdańsk University of Technology, Poland
}

\begin{abstract}
The paper deals with sensitivity and reliability applications to numerical studies of an off-shore platform model. Structural parameters and sea conditions are referred to the Baltic jack-up drilling platform. The sudy aims at the influence of particular basic variables on static and dynamic response as well as the probability of failure due to water waves and wind loads. The paper presents the sensitivity approach to a generalized eigenvalue problem and evaluation of the performace functions. The first order time-invariant problems of structural reliability analysis are under concern.
\end{abstract}

Keywords: Random variables, Structural reliability, Simulation methods, Offshore structures

\section{INTRODUCTION}

Off-shore platforms belong to the group of structures that work in strong technological and environmental regimes. Methods of probabilistic engineering mechanics applied in the analysis of these structures seem to be the most versatile [1]. This observation comes from the non-deterministic nature of the sea environment, exposing the platform structures to extreme sea waves, wind, current, seismic actions, ice, loads, ship collisions, technological impacts due to drilling works, etc. The requirements of severe environmental conditions and growing expectations of strong offshore industry contribute to the development of various structural systems, consequently invented, designed and operated on the basis of safety factor idea [2]. Valuable views on different offshore engineering problems can be found in [3], [4], [5], [6], [7], [8] and [9].

The paper presents a numerical approach to sensitivity and reliability problems of the Baltic steel drilling platform. This is a jack-up type platform, originally intended to operate under the Baltic Sea (Fig. 1). In a thirty-year period the platform has been involved in intensive exploration within the Polish Baltic zone, contributing in the development of oil and gas industry in Poland.

Key structural action has been recently undertaken for the structural rearrangement of the platform in order to implement its new drilling stationary functions.

In the analysis of offshore structures there are many engineering problems of high importance, e.g. the structuresea-subsoil interactions, determination of hydrodynamic, aerodynamic and seismic loads, determination of subsoil parameters, fatigue analysis, safety evaluation, etc. Due to the complexity of the mentioned problems no exact solutions are available, therefore, in order to asses the risk of failure of offshore platforms, approximate procedures must be used. In the paper structural sensitivity theory, e.g. [10], [11] and structural reliability analysis [1], [12] are applied. 


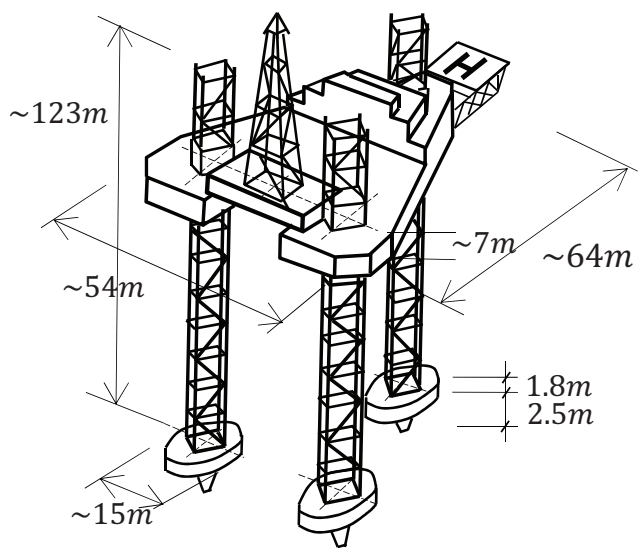

Fig. 1. General view of the Baltic drilling platform (dimensions in meters)

A numerical example deals with sensitivity and reliability computations due to parameters of the Baltic drilling platform [13], [14]. Thus it is the example of real engineering tasks with structural specifications of an existing system.

Sensitivity analysis of the structural response (i.e. displacements, internal forces, reactions, free vibration frequency of space frames and trusses) was performed due to variations of the basic (design) variables [15] was performed. Global structural geometry, cross-sectiona dimensions, material constants, support spring constants as well as external loads define basic variables. The computer program for statics and harmonic vibrations of space frames was developed. The sensitivity approach makes it possible to formulate performance functions (failure functions) further applied in reliability analysis. The first order approximation of failure probability is linearization of the performance surface in the domain of standard normal variables, i.e. replacing it by a hyperplane. The design point on the limit-state surface is the point of minimum distance to the origin. Two methods are used in searching the design point, i.e. the method known as the HL-RF method [16] and the directional simulation method, where failure probability in each sample is computed conditionally in a direction in the standard normal space [17] and [18]. These two methods are employed in the author software (cf. [19]).

\section{STRUCTURAL MODEL}

The platform is discretized by means of the Finite Element Method [20]. Linear space beam or truss models can be used. Structural parameters (i.e. geometry of the structure, crosssectional dimensions, length of elements, material constants and support spring constants) and the external loads are considered basic variables. Reliability analysis employs random modelling of the external loads and the subsoil parameters. Loads are assumed point forces with random intensities acting at nodes of the discrete model. Statistical parameters of the load intensity distribution, i.e. the mean value and standard deviation are defined on the basis of static solution and spectral properties of the load process ( the spectral approach was used in the papers of Jesień, 1987 and Rozmarynowski, 1990). The performance functions, created with the help of sensitivity-directed software, express either maximum horizontal deck displacement or flexural loadbearing capacity of the beam cross-section.

\section{STRUCTURAL DATA}

Figure 2 presents the discrete model with 72 degrees of freedom and all structural, subsoil, wave and wind parameters used in the analysis [13].

\section{DESCRIPTION OF LOADS}

Specification of random loads, i.e. names, distribution types, mean values and standard deviations of the corresponding random variables are shown in the Table 1. The subscript in each variable indicates the appropriate node number due to FEM discretization.

The mean values of particular random variables are evaluated on the basis of static analysis of the Baltic platform [21]. The standard deviations of the wind and wave loads in the considered case are computed using spectral characteristics of the load processes (cf. [13] and [14]. The coefficient of variation of the self-weight was assumed 10\%. Fig.2 incudes the following wave field data parameters: $\bar{H}, \bar{T}, \bar{L}$, are mean height, mean period and mean length for long-crested waves, respectively; $C_{M}^{\prime}, C_{M}^{\prime \prime}, C_{D}$ are empirical inertia and drag coefficients of the Morison equation [22].

In the case of slender enough and vertical cylinders, fixed in the sea bottom, the equation takes the form:

$$
\begin{aligned}
& \mathrm{d} \mathbf{P}_{H}(x, \mathrm{t})=\mathrm{d} \mathbf{P}_{H I}(x, \mathrm{t})+d \mathbf{P}_{H D}(x, t)= \\
& \rho \frac{\pi d^{2}}{4}\left\{\left[C_{M}^{\prime} \frac{\partial \mathbf{v}_{n}(x, t)}{\partial t}+C_{M}^{\prime \prime}\left(\frac{\partial \mathbf{v}_{n}(x, t)}{\partial t}-\frac{\partial \dot{\mathbf{q}}_{n}(x, t)}{\partial t}\right)\right]\right. \\
& \left.+\frac{1}{2} d C_{D}\left(\mathbf{v}_{n}(x, t)-\dot{\mathbf{q}}_{n}(x, t)\right)\left|\mathbf{v}_{n}(x, t)-\dot{\mathbf{q}}_{n}(x, t)\right|\right\} d x
\end{aligned}
$$

where $d \mathbf{P}_{H}(x, t)$ is the wave force (the hydrodynamic load vector) normal to the local axis $O x$ of the cylinder of a length $d x$. This force is aligned with the direction of wave propagation. $d \mathbf{P}_{H I}$ is the inertia force and $d \mathbf{P}_{H D}$ denotes the drag force; $\rho$ is the density of water; $d$ is the diameter of the submerged cylinder; subscript $n$ denotes "normal" to the cylinder axis; $\mathbf{v}(x, t)$ stands for the water particle velocity in the undisturbed wave field; $\dot{\mathbf{q}}(x, t)$ defines the cylinder response (velocity); $C_{M}^{\prime \prime}$ is the added mass coefficient - part of the Eqn. (1), involving the cylinder acceleration, complements suitable terms of the structural mass matrix; $\partial / \partial t$ denotes the partial derivative.

The last term of the Morison Eqn. (1) is non-linear, it represents the hydrodynamic drag forces. This term was studied in detail in [14]. If the analysis covers static (and free undamped vibration) computations, the following terms from Eqn. (1) are required: 


$$
\begin{aligned}
& \mathbf{P}_{H I}^{i}=\int_{-l / 2}^{l / 2} d \mathbf{P}_{H I}^{i}=C_{M} \frac{\rho g}{l} \mathbf{V}_{i} H K_{I} \\
& \mathbf{P}_{H D}^{i}=\int_{-l / 2}^{l / 2} d \mathbf{P}_{H D}^{i}=\frac{1}{2} C_{D} \frac{\rho g}{l} \mathbf{A}_{i} H^{2} K_{D}
\end{aligned}
$$

where $l$ is the element length; $g$ stands for the acceleration of gravity; $\mathbf{V}_{i}$ denotes the volume of the submerged element associated with the $i$-th node of the discrete model; $H$ is the wave height and:

$K_{I}=\frac{1}{2} k \int_{-l / 2}^{l / 2} \frac{\cosh (k(x+h))}{\cosh (k h)} \sin \left(-\frac{2 \pi t}{T}\right) d x$

where $k=2 \pi / L, h$ is the depth of the sea, $x$ indicates the local axis variable, $T$ denotes the wave period; $\mathbf{A}_{i}$ is the projected area of the submerged element:

$K_{D}=\frac{1}{4} k \int_{-l / 2}^{l / 2} \frac{\cosh ^{2}(k(x+h))}{\sinh (k h) \cosh (k h)}\left|\cos \left(\frac{2 \pi t}{T}\right)\right| \cos \left(\frac{2 \pi t}{T}\right) d x$

The engineering applications of Eqns. (2) direct the investigation to extreme values. Additionally, these extremes do not occur simultaneously. The appropriate factor to reduce their combination depends on the ratio $C_{M} d / C_{D} H$. The inertia and drag forces obtained this way (after integration from the sea bottom to the free sea elevation, with respect to the length of the beam finite elements) results in the load mean values.

$D_{Z}$ specified in Fig. 2 is an equivalent diameter of the platform leg composed of four cylinders, each of diameter $d$.

Wind field data parameters (Fig. 2): $\bar{V}_{10}$ is the mean speed at a height of $10 \mathrm{~m}$ above the surface; $\chi$ denotes the surface drag coefficient, $L$ is the length scale of turbulence, $a, b, c$ are empirical coefficients in the Davenport's formula expressing the cross-spectral density of the along-wind fluctuating velocity [23], $C_{D A}$ is the drag coefficient .

\section{THE SUBSOIL PROPERTIES}

The subsoil parameters are assumed deterministic or random. In both variants the subsoil is assumed a homogeneous linear half-space defined by shear modulus $G$ and Poisson's ratio $v$. It is assumed that in the second variant of the subsoil model, shear modulus is random, whereas Poisson's ratio is deterministic. The soil-structure interaction is simulated by a system of springs. The mean values and the variances of the spring coefficients are [13], [24]:

$$
\begin{aligned}
& K_{i X}^{H}=K_{i Y}^{H}=\frac{8 r_{0}}{2-v} E(G), K_{i Z}^{V}=\frac{4 r_{0}}{1-v} E(G), \\
& K_{i X}^{R}=K_{i Y}^{R}=\frac{8 r_{0}^{3}}{3(1-v)} E(G), K_{i Z}^{T}=\frac{16 r_{0}^{3}}{3} E(G) \\
& \sigma_{K_{H}}^{2}=\frac{64 r_{0}^{2}}{(2-v)^{2}} \sigma_{G}^{2}, \sigma_{K_{V}}^{2}=\frac{16 r_{0}^{2}}{(1-v)^{2}} \sigma_{G}^{2}, \\
& \sigma_{K_{R}}^{2}=\frac{7.11 r_{0}^{6}}{(1-v)^{2}} \sigma_{G}^{2}, \sigma_{K_{T}}^{2}=28.44 r_{0}^{6} \sigma_{G}^{2}
\end{aligned}
$$

where the superscripts $H, V, R$ and $T$ denote horizontal, vertical, flexural and torsional components of the spring stiffnesses, respectively; $r_{0}$ is the radius of the disc, $E(\bullet)$ denotes mathematical expectation and $\sigma_{G}^{2}$ is the variance of shear modulus $G, i$ stands for the node number at the bottom. It results in the values shown in Table 2 .

STRUCTURAL PARAMETERS:

1. Element stiffnesses

\begin{tabular}{lccc}
\hline Part & $E A[M N]$ & $G J_{s}\left[\mathrm{MNm}^{2}\right]$ & $E J\left[\mathrm{MNm}^{2}\right]$ \\
\hline Deck & $8.40 \cdot 10^{5}$ & $1.94 \cdot 10^{6}$ & $2.53 * 10^{6}$ \\
Legs & $0.79 * 10^{5}$ & $6.86 * 10^{5}$ & $8.93 * 10^{5}$ \\
\hline
\end{tabular}

2. Lumped masses (translational)

\begin{tabular}{cc}
\hline Node & Mass $[\mathrm{Mg}]$ \\
\hline $1(2,3)$ & 300 \\
$4(5,6)$ & 1800 \\
$7(8,9)$ & 310 \\
$10(11,12)$ & 160 \\
Total & $2570 \times 3=7710$ \\
Hydrodynamic masses & 550 \\
Deck exploitation mass & 1700 \\
\hline
\end{tabular}

WAVE FIELD PARAMETERS:

$\bar{H}=14 m, \bar{T}=10 \mathrm{~s}, \bar{L}=180 \mathrm{~m}$

$C_{M}^{\prime}=C_{M}^{\prime \prime}=C_{D}=1$

WIND FIELD PARAMETERS:

$\bar{V}_{10}=40 \mathrm{~m} / \mathrm{s}, L=1200 \mathrm{~m}, \chi=0.005, a=b=c=1, C_{D A}=1$,

$D_{z}=4 m$ 


\section{SENSITIVITY OF THE EIGENVALUE PROBLEM}

The solution to the generalised eigenproblem of the model in Fig. 2 provides to the following natural frequencies (see Table 4). The sensitivity analysis deals with the first natural frequency and the corresponding eigenmode, this is presented in Fig. 3

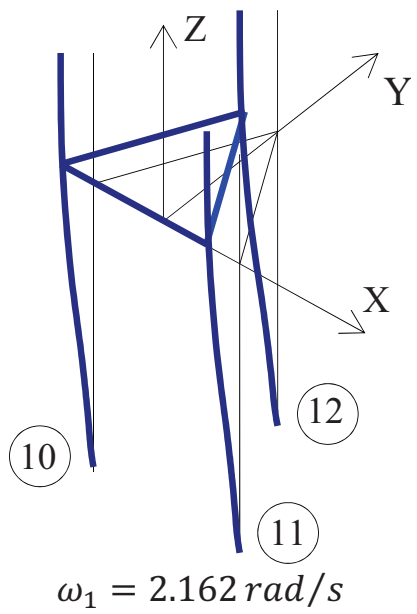

Fig. 3. The analysed first eigenfrequency and eigenmode

In the present example the support spring constraints in nodes 10,11 i 12 were taken into account as design variables. The variation of natural frequency is as follows

$$
\frac{\delta \omega_{1}}{\omega_{1}}=\sum_{j=1}^{n} W_{l j} \frac{\delta x_{j}}{x_{j}}
$$

where $W_{l i}$ stands for the first-order sensitivity coefficient of the design variable $x_{j}$. The sensitivity coefficients for the spring stiffnesses at the indicated nodes are collected in Table 3.

According to Fig. 3 the first eigenmode, shows flexural deformation type along the OX axis, the sensitivity coefficients are presented in Table 3 for perpendicular directions, i.e. $K_{Y}$ (nodes $10,11,12$ ) and $K_{Z}$ (node 12), as well as for rotations along the $\mathrm{OX}$ axis, i.e. $K_{\varphi X}$ and the $\mathrm{OZ}$ axis, i.e. $K_{\varphi \mathrm{Z}}$, are equal zero.

The framed sensitivity coefficients in Table 3 reveal the influences of rotational and translational springs, i.e. $K_{\varphi Y}$ and $K_{Z}$, on variations of the first eigenfrequency and eigenmode. The obtained results are drawn in Fig. 4 with respect to the node no 10. Similar results were obtained for the remaining support nodes, i.e. no 11 and no 12.

Aditionally, Fig. 4 shows that the eigenmode sensitivity is highly affected by translational spring variation, the impact of the rotational one is lower. This is a key practical conclusion, while one of the platform legs meets a weak subsoil layer, a consequent, sudden break may cause structural instability.

Limitting the set of design variables to these related to the vertical components of spring supports in nodes 10,11 and
12 in terms of the assumed variations, i.e. $\delta K_{10 z}=0.2 K_{10 z}$ , $\delta K_{11 Z}=-0.2 K_{10 Z}, \delta K_{12 Z}=0.1 K_{10 Z}$, the comparative sensitivity computations are depicted in Fig. 5.

The direct eigenmode solution indicated in Fig. 5 is an alternative approach used in the study to compare the sensitivity results.

Generally, the first-order variations of the structural response (i.e. displacements, cross-sectional forces, support reactions, free vibration frequencies) due to variations of the basic (design) variables (i.e. loads, Young's modulus, Poisson's ratio, mass density constant, sectional shear distribution factor or cross-sectiona dimensions) can be analysed with the help of the author software developed for statics and harmonic vibrations of space frames.

\section{RELIABILITY ANALYSIS}

In the reliability analysis assumptions upon the performance functions are required. In the paper these functions are assumed to take the following two conditions:

Displacement barrier (stiffness condition):

$$
g_{1}\left(d_{p}, \mathbf{X}\right)=1-\frac{q(\mathbf{X})}{d_{p}}
$$

where $\mathbf{X}^{T}$ is the vector of random variables including: vave and wind loads described in Table 1 , where $v_{X_{i}}$ denotes the coefficient of variation of the random variable $X_{i}$, selfweight loads modelled by the normal distribution with the mean values computed with the help of lumped masses data presented in Fig. 2 and the coefficient of variation equal 10\% and spring stifnesses replaced by the normal distribution with the mean values shown in Table 2 and the coefficient of variation $50 \%, q(\mathbf{X})$ denotes the response (displacement) function, $d_{p}$ is the maximum allowable drift of the platform.

Cross-section capacity condition:

$$
g_{2}\left(M_{p}, N_{p}, \mathbf{X}\right)=1-\left\{\left[\frac{M(\mathbf{X})}{M_{p}}\right]^{\alpha_{1}}+\left[\frac{N(\mathbf{X})}{N_{p}}\right]^{\alpha_{2}}\right\}
$$

in which $\alpha_{1}$ and $\alpha_{2}$ are constant coefficients, $M(\mathbf{X})$ and $N(\mathbf{X})$ are the bending moment and axial force, $M_{p}=W R_{p l}$, $N_{p}=A R_{p l}$, where $W$ and $A$ are section modulus and crosssection area, $R_{p l}$ is the yield stress. The parameters of the performace functions in Eqs. (5) and (6), i.e. $d_{p}, W, A$ and $R_{p l}$ can be generally considered random variables.

In the example the yield stress is assumed Gaussian random variable of the following statistical characteristics:

$$
\begin{aligned}
& E\left(R_{p l}\right)=250 \mathrm{MPa}, \\
& \sigma_{R_{p l}}=0.125 \cdot E\left(R_{p l}\right)=31.25 \mathrm{MPa}
\end{aligned}
$$


A

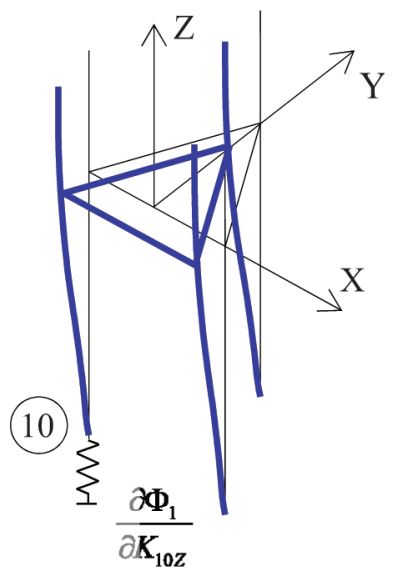

B

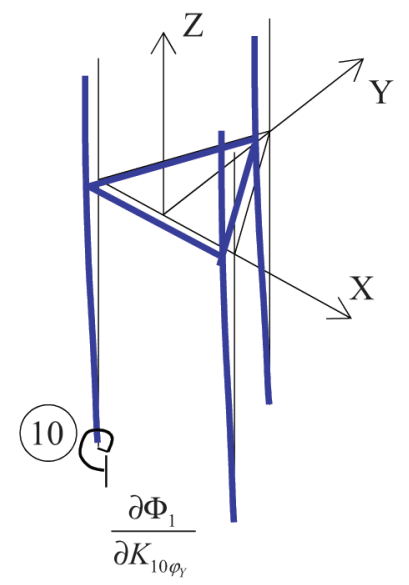

Fig. 4. Sensitivity coefficients of the first eigenmode for the node $\mathrm{Nr} 10$

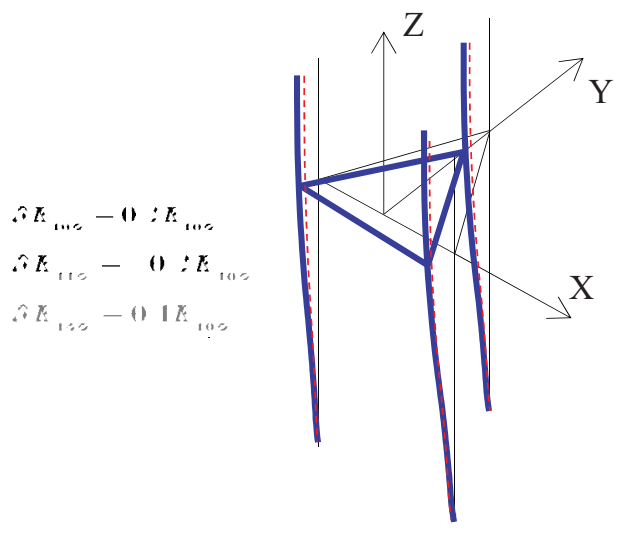

Sensitivity analysis

Direct eigenmode solution

Fig. 5. Comparison of the results

The remaining parameters are taken arbitrarily, based on structural data, i.e. $W=1.27 \mathrm{~m}^{3}, A=0.38 \mathrm{~m}^{2}$ and $\alpha_{1}=\ddot{\mathrm{u}} \alpha_{2}=$ (cf. [25]).

Assume that $S(\mathbf{X})$ is the following general function of the response variables, i.e. $S(\mathbf{X})=q_{6 Y}(\mathbf{X})$ in Eq. (5), where $q_{6 Y}(\mathbf{X})$ is the horizontal displacement in Y-direction at the deck node Nr 6 (Fig. 2) and $S(\mathbf{X})=\{M(\mathbf{X}), N(\mathbf{X})\}$ in Eq. (6). Let Taylor's series expansion of $S(\mathbf{X})$ exist at the mean value point $\mu_{\mathbf{X}}=E(\mathbf{X})$ :

$$
\begin{aligned}
& S(\mathbf{X})=S\left(\mu_{\mathbf{X}}\right)+\sum_{i=1}^{n}\left(\frac{\partial S}{\partial X_{i}}\right) \cdot\left(X_{i}-\mu_{X i}\right)+\Psi(\mathbf{X})= \\
& =a_{0}+\sum_{i=1}^{n} a_{i} X_{i}+\Psi(\mathbf{X})
\end{aligned}
$$

where $\Psi(\mathbf{X})$ represents the higher-order terms.

The coefficients in Eq. (7) can be computed in several ways including direct differentiation method, iterative perturbation algorithms [26], [27] and sensitivity methods, i.e. the adjoint method [10] with the use of finite-element procedures. In the paper the sensitivity approach was applied. It yields the statement that the influence of the self-weight variability of loads on the structural response is negligible. It means that the space of basic random variables can be reduced to those representing wind and wave loads and spring stiffnesses. It is assumed that random variables are uncorrelated or correlated.

A basic problem within structural reliability is to evaluate the probability of failure:

$$
P_{f_{i}}=P\left[g_{i}(\mathbf{X}) \leq 0\right]=\int_{g_{i}(\mathbf{x}) \leq 0} f(\mathbf{x}) d \mathbf{x}
$$

where $f(\mathbf{x})$ stands for the joint probability density function of $\mathbf{x} \in \mathbf{X}, g_{i}(\mathbf{X}), i=1,2$, are the performance functions formulated such that $g_{i}(\mathbf{X}) \leq 0$ defines failure of the structure and $g_{i}(\mathbf{X})>0$ denotes its survival. To set up the joint probability distribution the Nataf's multivariate distribution model with determined marginal distributions and covariances between them was implemented [28].

The main results of the reliability computation for the platform numerical model presented in Fig. 2 are given in Table 5 and Table 6, where First Order Reliability Method (FORM) and Second Order Reliability Method (SORM) were employed. Probabilities of separate failure modes are presented for uncorrelated and correlated random variables representing wind and wave loads. The maximum allowable displacement was assumed $d_{p}=0.5 \mathrm{~m}$.

The HL-RF method originally proposed by Hasofer and Lind and extended by Rackwitz and Fiessler was applied in the presented analysis because experience shows [16] that this method requires small amounts of storage and computation in each step, moreover, in most situations this method converges rapidly.

The Monte Carlo directional simulation method (MCDS) was applied herein in order to compare the results of the HL-RF method. The method is generally applicable for timeinvariant reliability integration. The importance sampling technique used in directional sampling in the case of convex polyhedral failure [17] set results in considerably reduced number of trials. In the analysed numerical example the number of trials did not exceed 1000. 


\section{CONCLUDING REMARKS}

The paper presents the first-order sensitivity and timeinvariant reliability approaches of the Baltic drilling platform subjected to wind and wave loads. On the basis of the analysis, it is possible to formulate the following conclusions.

1. All random variables do not act equally on structural reliability. Sensitivity methods may be practically used to quantify the influence of each basic random variable. In the numerical example presented, the self-weight took a negligible influence on response variations. Thus the space of basic random variables was considerably reduced.

2. The data in the numerical example made the displacementbased performance function (stiffness condition) decisive in the structural safety assessment.

3. The probability of the first failure mode increases when the correlation coefficient between the wind and wave loads increases. An opposite tendency one can observe in the second failure mode.

5. Greater probabilities of failure were obtained when the subsoil model has random parameters (cf. Table 6 and [29]).

6. In the considered problem the directional simulation method worked well, compared to the HL-RF method. The limit of 1000 trials was sufficient to achieve accepted convergence. Simulations by classical Monte Carlo method required ten times more trials.

\section{REFERENCES}

1. Chandrasekaran S.: Offshore structural engineering. Reliability and risk assessment. Taylor and Francis Group, 2016.

2. Chakrabarti S.K.: Handbook of offshore engineering. Vol. 1, 2, Elsevier, 2005.

3. Chakrabarti S. K.: Nonlinear Methods in Offshore Engineering, Developments in Marine Technology, 5, Elsevier, 1990.

4. Faltinsen O.M.: Sea loads on ships and offshore structures. Cambridge University Press, 1990.

5. Clauss G., Lehmann E., Ostergaard C.: Offshore structures. Vol. 1, 2. Springer, 1992.

6. Gran S.: A Course in Ocean Engineering. Developments in Marine Technology, Elsevier, 1992.

7. Moan T. Reliability and Risk Analysis for Design and Operations Planning of Offshore Structures. Structural Safety and Reliability, ICOSSAR'93, 1, Balkema, Rotterdam, 1994, pp.21-43.

8. Wilson J.F.: Dynamics of offshore structures. John Willey \& Sons, 2003.
9. Bai Y., Bai Q.: Subsea engineering handbook. Elsevier, 2012.

10. Haug E. J., Choi K. K. and Komkov V.: Design Sensitivity Analysis of Structural Systems. Academic Press, New York, 1986.

11. Szymczak Cz..: Elementy teorii projektowania (in Polish). PWN, Warszawa 1988.

12. Melchers R.E.: Structural reliability and predictions. John Wiley \& Sons, Chichester, 1999.

13. Jesien W.: Random Vibrations of the Baltic Drilling Platform Subjected to Wind Loads and Water Waves. Earthquake Engineering and Structural Dynamics, 15, 1987, pp. 595-617.

14. Rozmarynowski B.: Averaged Damping in Random Vibrations of the Baltic Drilling Platform. Journal of Sound and Vibration 139(3), 1990, pp. 437-458.

15. Mikulski T. and Szymczak Cz.: Sensitivity Analysis of Free Vibration Frequencies of Frames including Shear Rotary Inertia Effects. Proceedings of XI International Conference on Computer Methods in Mechanics, SzczecinSwinoujœcie, 14-20 May 1990.

16. Liu P.-L. and Der Kiureghian A.:. Optimization Algorithms for Structural Reliability. Structural Safety, 9, 1991, pp. 161-177.

17. Bjerager P.: Probability Integration by Directional Simulation. Journal of Engineering Mechanics, ASCE, 114(8), 1986, pp.1285-1302.

18. Shao S. and Murotsu Y.: Reliability of Complex Systems Using an Efficient Directional Simulation. Structural Safety and Reliability, ICOSSAR'93, 3, Balkema, Rotterdam, 1994, pp.1529-1534.

19. Liu P.-L., Lin H.-Z., Der Kiureghian A.: CALREL user manual. Report No. UCB/SEMM-89/18, Berkely 1989.

20. Jesień W., Rozmarynowski B. and Wizmur W.: Dynamical response of the Baltic drilling platform loaded by wind waves and wind. Proceedings of an International Conference on Computer Modelling in Ocean Engineering, Venice, 19-23 Sept., Balkema, Rotterdam, 1988, pp. 531-537.

21. Wizmur M., Jesień W. and Rozmarynowski B.: Static and dynamic algorithms for the Baltic platform structure deterministic approach. Internal working report, (in Polish), Gdańsk, 1981.

22. Morison, J.R., O’Brien, M.P., Johnson, J.W. and Schast, S.A.: The forces excerted by surface waves on piles. PetroleumTrans., ASME, 189, 1950,pp. 149-154. 
23. Davenport A. G.: The Spectrum for Horizontal Gustiness near the Ground in High Winds. Quarterly Journal of the Royal Meteorological Society, 87, 1961, pp.194-211.

24. Clough R.W. and Penzien J.: Dynamics of Structures. McGraw-Hill, New York, 1975.

\section{CONTACT WITH THE AUTHORS}

25. Sokol L.: Sollicitations ultimes dans une section circulaire. Construction Metallique (in French). No. 2, 1988, pp. 73-80.

26. Dias J., Nagtegaal J. and Nakazawa S.: Iterative perturbation algorithms in probabilistic finite analysis. Computational Mechanics of Probabilistic and Reliability Analysis, Elmepress International, Virginia, 1989, pp. 211-230.

\section{Bogdan Rozmarynowski Tomasz Mikulski}

Gdańsk University of Technology 11/12 Narutowicza St. 80-233 Gdańsk

Poland

27. Wu Y.-T., Millwater H. R. and Cruse T. A.: Advanced probabilistic structural analysis method for implicit performance functions. AIAA Journal, 28(9), 1990, pp. 1663-1669.

28. Liu P.-L. and Der Kiureghian A.: Multivariate distribution models with prescribed marginals and covariances. Probabilistic Engineering Mechanics, 1(2), 1986, pp. 105-112.

29. Rozmarynowski B.: Reliability analysis of a stochastically non-linear structural system. Proceedings of the International Conference on Safety and Reliability, ESREL'97, Vol. 2, Lisbon, Portugal, 1997, pp. 1509-1517.

30. Breitung K.: Asymptotic approximations for multinormal integrals. Journal of Engineering Mechanics, ASCE 110(3), 1984, pp. 357-366. 
Tab. 1. Random variables $X_{i}$ and their statistical parameters $X_{0 i}, v_{X i}$

\begin{tabular}{|c|c|c|c|c|}
\hline$X_{i}$ variable $^{1}$ & Name & Distribution & $X_{0 \mathrm{i}}=E\left({ }_{X i}\right)[\mathrm{kN}]$ & $v_{X i}$ \\
\hline$Y_{1}, Y_{2}, Y_{3}, Y_{4}, Y_{5}, Y_{6}$, & Wind load & Gumbel & 280.6 & 1.25. \\
\hline$Y_{7}, Y_{8}, Y_{9}$, & Wave load & Log-normal & 446. & 2.60 \\
\hline$Y_{10}, Y_{11}, Y_{12}$, & Wave load & Log-normal & 290.6 & 2.60 \\
\hline
\end{tabular}

Tab. 2. Mean values of stifnesses of the suport springs

\begin{tabular}{|l|c|}
\hline \multicolumn{1}{|c|}{ Type of the spring displacement } & Stifness \\
\hline Translational horizontal $K_{i X}, K_{i Y}[\mathrm{Mn} / \mathrm{m}]$ & 783.3 \\
\hline Translational vertical $K_{i Z}[\mathrm{Mn} / \mathrm{m}]$ & 1044.4 \\
\hline Rotational horizontal $K_{i X}^{R}, K_{i Y}^{R}[\mathrm{Mn} / \mathrm{m}]$ & 37204.2 \\
\hline Rotational vertical $K_{i Z}^{R}[\mathrm{Mn} / \mathrm{m}]$ & 44645.0 \\
\hline
\end{tabular}

Tab. 3 Sensitivity coefficients of the eigenfrequency

\begin{tabular}{cccc}
\hline Spring constraint & Node 10 & Node 11 & Node 12 \\
\hline$K_{X}$ & 0.00589 & 0.00589 & 0.00457 \\
$K_{Y}$ & 0.00003 & 0.00003 & 0.00000 \\
$K_{Z}$ & 0.05536 & 0.05536 & 0.00000 \\
$K_{\varphi X}$ & 0.00015 & 0.00015 & 0.00000 \\
$K_{\varphi Y}$ & 0.10694 & 0.10694 & 0.09590 \\
$K_{\varphi Z}$ & 0.00000 & 0.00000 & 0.00000 \\
\hline
\end{tabular}

Tab. 4. Natural frequencies of the model with elastic supports.

\begin{tabular}{|c|c|c|c|}
\hline$\omega_{1}=2.162 \mathrm{rad} / \mathrm{s}$ & $\omega_{2}=2.163$ & $\omega_{3}=3.445$ & $\omega_{4}=9.434$ \\
\hline
\end{tabular}

Tab. 5. Probabilities of failure modes, $d_{p}=0.5 \mathrm{~m}$, deterministic spring stiffnesses. FORM

\begin{tabular}{|c|c|c|c|c|}
\hline \multirow{2}{*}{ Method } & \multicolumn{3}{|c|}{ Correlation coefficient between wind and wave loads } \\
\hline & $P_{f 1}$ & 0.0 & $P_{f 2}$ & 0.20 \\
\cline { 2 - 5 } & $1.413 \cdot 10^{-3}$ & $1.693 \cdot 10^{-5}$ & $2.550 \cdot 10^{-3}$ & $1.593 \cdot 10^{-5}$ \\
\hline HL-RF. & $1.585 \cdot 10^{-3}$ & $1.823 \cdot 10^{-5}$ & $2.879 \cdot 10^{-3}$ & $1.691 \cdot 10^{-5}$ \\
\hline MCDS $^{2}$ & &
\end{tabular}

Tab. 6. Probabilities of failure modes, $d_{p}=0.5 \mathrm{~m}$, random spring stiffnesses. FORM/SORM

\begin{tabular}{|c|c|c|c|c|}
\hline Method & \multicolumn{3}{|c|}{ Correlation coefficient between wind and wave loads } \\
\hline & \multicolumn{2}{|c|}{0.0} & $P_{f 2}$ & 0.20 \\
\hline & $P_{f 1}$ & $1.593 \cdot 10^{-3}$ & $4.055 \cdot 10^{-3}$ & $1.530 \cdot 10^{-3}$ \\
\hline HL-RF & $2.343 \cdot 10^{-3}$ & $1.572 \cdot 10^{-3}$ & $4.379 \cdot 10^{-3}$ & $1.394 \cdot 10^{-3}$ \\
\hline MCDS and IB
\end{tabular}

\section{Footnotes:}

1 Subscript indices of the variables reffer to the node $\mathrm{Nr}$ indicated in Fig. 2 .

MCDS denotes Monte Carlo Directional Simulation method.

3 The probabilities $P_{f 2}$ were obtaineed by applying improved Breitung (IB) formula [30]. 